\title{
Study on Stress Analysis and Experimental Measurement of Isolated Switch Porcelain Insulator
}

\author{
Peng Wang ${ }^{1}$, Qiaodi Zeng ${ }^{1}$, Weixing Liao ${ }^{1}$, Cheng $\mathrm{Ma}^{2}$, Hui Peng ${ }^{2, \text { a, }{ }^{*}}$ \\ ${ }^{1}$ Shenzhen Power Supply Bureau Co., Ltd., Shenzhen, China \\ ${ }^{2}$ School of Electrical Engineering Wuhan University, Wuhan, China \\ ahpeng@whu.edu.cn
}

\begin{abstract}
Keywords-post porcelain insulator; fracture accident; tractive force of bus; stress distribution; finite element method(FEM).

Abstract. The post porcelain insulator is pulled by bus, which will produce stress in post porcelain insulator, and stress concentration is in the root of the umbrella. Therefore, there will be break accident if it is not installed properly. Firstly, the fracture theory of porcelain insulator is summarized from the characteristics of ceramic materials, including its way to fracture and the characteristic quantities emerged when fracturing. Then the brittle fracture theory of the maximum tensile stress theory of the porcelain insulator is summarized. The ANSYS based on finite element method is used for simulation analysis. The finite element model of different types of porcelain insulators is established, the stress distribution of the porcelain post insulator in different directions and of different sizes are calculated. The maximum stress value of the structure of the umbrella skirt is changed correspondingly by changing the direction and size of the force of the bus line. The relationship between stress and load force mainly presents a linear relation with load. According to the calculation of the stress distribution of the porcelain insulator using finite element method, the relationship curves of stress and load direction and magnitude are plotted, and the theoretical results are verified.
\end{abstract}

\section{Introduction}

As one of the important electrical equipment in the transformer substation, the post insulator (porcelain insulator) plays a double role of insulation and support. Therefore its safe and stable operation is directly related to the stability and reliability of the substation, but also related to the safety of the operator. The porcelain vase column is widely used in today's substation, such as switches, transformers, GIS lines and so on. Whether porcelain column can operate reliably and stably or not will influence the security of network. There are power accidents caused by the fracture of the porcelain insulator porcelain column every year, which will cause the damage of isolated switches even the short-circuit of substation bus, endangering the safe operation of the whole system.

The relevant data ${ }^{[1]}$ shows that the fracture accident of the porcelain insulator is mainly divided into the broken and cracked porcelain column, in which the broken porcelain column of the accident accounted for $36 \%$, cracking accident accounted for $62 \%$. In addition, the ratio of the broken porcelain insulator which is used to isolate switches and support the bus bar accounted for $95 \%{ }^{[2]}$. And from the fracture site of insulator, the occurrence of the fault location is in the lower part of the accident, on the central part of the occurrence.

Porcelain insulator usually can be divided into line porcelain insulator, power station porcelain insulator ${ }^{[3]}$ according to the its application. The power station porcelain insulators mainly adopt the porcelain insulator, which can be used in the isolation switch, bus, grounding switch and the stop wave device in the substation, playing an important role in insulation and mechanical fixation. The main insulation of each part of the porcelain post insulator is the umbrella skirt in the middle. It can be generally divided into three categories: conventional type, big-small umbrella type and prism under umbrella type. The distance between two umbrellas of big-small umbrella type insulation is large, having a good performance of rain flash and covering snow, while prism under umbrella type 
insulation allows a larger leakage distance, which makes the insulator have a higher pollution flashover voltage.

According to the fracture accident of the porcelain insulator, after studying a lot of reasons, the research will focused on the study of the material of porcelain insulator string. The ceramic material is a typical brittle material, brittle fracture is the result of many factors. For example, the improper installation of the insulator can cause the stress to be uneven, then comes the crack causing the brittle fracture. The poor working environment of the outside world will lead to uneven stress distribution in the insulator, resulting in stress concentration and brittle fracture. In this paper, the influence of other external environmental factors is neglected, which is aimed to study the reason of the brittle fracture of the porcelain insulator, considering the pulling force of the lead wire as the main reason.

\section{Basic Analysis of Fracture Theory of Porcelain Insulator}

\section{Characteristics of the fracture crack of porcelain insulator}

When a homogeneous plate with no macro cracks is subjected to tension, the stress at each point in the plate is equal to the average stress. When the plate containing cracks, as shown in the following figure, the crack tip region of the stress line density is increased with the increase of stress and crack length ${ }^{[4]}$. Stress intensity factor $\mathrm{K}$ is proposed in 1957 by G.R.Irwin ${ }^{[5]}$. Three stress field and strain field near the crack tip as shown in Fig 1 are described, the stress field distribution is summarized, and the crack propagation criterion with $\mathrm{K}$ as the characteristic quantity of is established with the analysis of the stress field near the crack tip. In this paper, only the open crack of type I will be analyzed.
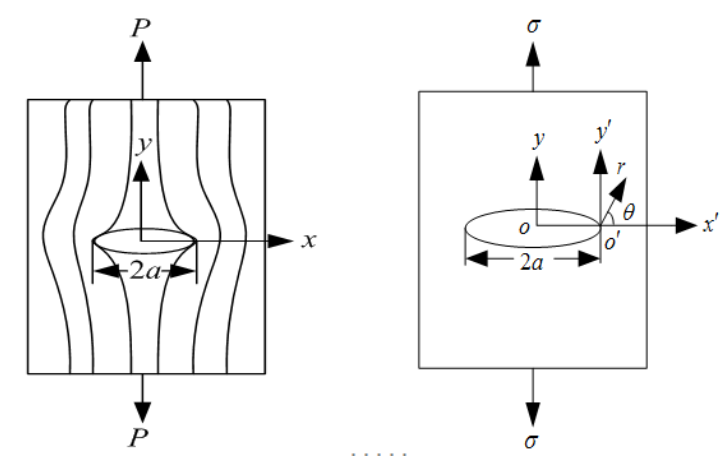

Fig. 1 Stress distribution and coordinate transformation of the open crack plate.

Stress field of the open crack can be expressed as:

$$
\begin{aligned}
& \sigma_{x}=\frac{K_{\mathrm{I}}}{\sqrt{2 \mathrm{r} r}} \cos \frac{\theta}{2}\left[1-\sin \frac{\theta}{2} \sin \frac{3 \theta}{2}\right] \\
& \sigma_{y}=\frac{K_{\mathrm{I}}}{\sqrt{2 \mathrm{r}}} \cos \frac{\theta}{2}\left[1+\sin \frac{\theta}{2} \sin \frac{3 \theta}{2}\right] \\
& \sigma_{z}=\frac{K_{\mathrm{I}}}{\sqrt{2 \mathrm{r} r}} 2 r \cos \frac{\theta}{2}
\end{aligned}
$$

Where, $K_{I}$ is stress field intensity factor near crack tip of type I, $r$ is the distance between a point and the origin of polar coordinates. Eq. 1-3 are approximate formula, the high-order of $r$ has been omitted. When the crack tip spacing is less than crack length, that is $r<<a$, the precision of the approximation is high enough, so it can be simplified as Eq. 4:

$$
\sigma_{x}=\sigma_{y}=\frac{K_{\mathrm{I}}}{\sqrt{2 \mathrm{~T} r}}, r<<a
$$


It can be conclude that, $K_{I}$ has nothing to do with $r, \theta$ in polar coordinates, is the state characteristics of stress field near the crack tip. For each point of the crack front, the coordinates $r$ are known, therefore the internal stress field is determined by $K_{I}$, and the relationship is almost linear. That is, the bigger the $K_{I}$ is, the greater the stress field corresponding to the stress field will be. Deform Eq. 1-4 as a general equation:

$$
K_{\mathrm{I}}=Y \sigma \sqrt{a}
$$

Where, $Y$ is related to the type of crack, the shape of the specimen and the mode of loading which is dimensionless. For wireless wide plate center penetrating crack, $\mathrm{Y}=\pi^{0.5}$.

It can be seen that stress field intensity factor $K_{I}$ is associated with $\sigma$ and crack length $a$. When the afterburner is increasing, $K_{I}$ is also increasing, the stress $\sigma$ will also increase, the final result in crack tip near the internal stress of $P$ will also be improved. When $K_{I}$ increases to a certain critical value, the critical fracture strength of $P$ is equal to or greater than that of brittle ceramic materials $P_{f}$. Then the material is separated from the tip of the crack, and the crack can be expanded rapidly, and the sample is brittle. The critical state of $K_{I}$ is called the critical intensity factor, expressed as $K_{I C}$, that is:

$$
K_{\mathrm{IC}}=Y \sigma_{C} \sqrt{a}
$$

Since $K_{I C}$ is a function of material structure, it reflects the inherent properties of the material, which is related to the material structure and composition, and is independent of the size, shape of the crack and the applied force, is the material internal crack resistance in the process of expansion, known as the material of the fracture toughness value. $\sigma_{C}$ is called as breaking strength correspondingly.

Then the general fracture of ceramic materials can be concluded that:

(1) $K_{I}<K_{I C}$, even the material contains the crack, but this crack will not lose its stability and brittle fracture under the condition of external stress.

(2) $K_{I} \geq K_{I C}$, the crack of the material in the external stress role will become unstable, and will eventually occur brittle fracture.

\section{Maximum tension stress theory}

The maximum tensile stress theory ${ }^{[6]}$, was first discovered and put forward by the Italy scholar G.Galilo in the strength test, which is called the first strength theory. The ultimate perfection of the theory is attributed to the famous British educator W.J.M.Rankine, so the theory is also called as Rankine's Theory ${ }^{[7]}$.

It is considered that when the material is under the influence of external stress, then stress distribution within the material itself will be produced according to the maximum tensile stress theory. Where the larger stress value of the point (area) is called the risk point (area), in the process of increasing the external stress, the stress value of the dangerous point (area) will also be increased, until the stress value is increased to the maximum tensile stress, and then brittle fracture of material will occur along the maximum tensile stress. Therefore, the maximum tensile stress theory holds that the reason for the brittle fracture of the material is that the tensile stress reaches or exceeds the tensile stress limit of the material. The corresponding relationship is expressed as Eq. 7:

$$
\sigma_{\max }=\sigma_{1} \geq \sigma_{0}, \sigma_{1}>0
$$

Where, $\sigma_{1}$ is tensile stress value of material under external stress, and $\sigma_{0}$ is material limit stress.

Through the analysis of the failure theory of porcelain insulator, the main reason of fracture is that the internal stress is too large. In this paper, the finite element analysis method is used to simulate the stress of porcelain insulator. 
The general steps of finite element analysis and the study of the relevant theory of stress field

The finite element analysis method, as a mature method, has formed a fixed step. ANSYS software for the analysis has also formed a fixed step, generally divided into 7 steps, that is:

(1)Select the appropriate unit and give the unit real constant;

(2)Set material properties;

(3)Build model;

(4)To give the corresponding model to the material properties, and to set up the appropriate unit size to divide the grid unit;

(5)Impose boundary conditions;

(6)Finite element calculation results;

(7)Post-processing operation, get the required data and results.

As the finite element analysis method is presented in the structure analysis ${ }^{[8]}$, and it is most widely used in the field of mechanics. For mechanical problems, in fact, is a problem of force field analysis of Orlando. Corresponding to each field, there are corresponding control equations, the stress field in the control equation is shown below:

(1) Equilibrium differential equation:

$$
\left.\begin{array}{c}
\frac{\partial \sigma_{x}}{\partial x}+\frac{\partial \tau_{x y}}{\partial y}+\frac{\partial \tau_{x z}}{\partial z}+f_{x}=0 \\
\frac{\partial \tau_{y x}}{\partial x}+\frac{\partial \sigma_{y}}{\partial y}+\frac{\partial \tau_{y z}}{\partial z}+f_{y}=0 \\
\frac{\partial \tau_{x z}}{\partial x}+\frac{\partial \tau_{y z}}{\partial y}+\frac{\partial \sigma_{z}}{\partial z}+f_{z}=0
\end{array}\right\}
$$

(2) Geometric equation:

$$
\left.\begin{array}{l}
\varepsilon_{x}=\frac{\partial u}{\partial x} ; \gamma_{x y}=\frac{\partial u}{\partial y}+\frac{\partial v}{\partial x} \\
\varepsilon_{y}=\frac{\partial v}{\partial y} ; \gamma_{y z}=\frac{\partial v}{\partial z}+\frac{\partial w}{\partial y} \\
\varepsilon_{z}=\frac{\partial w}{\partial z} ; \gamma_{z x}=\frac{\partial w}{\partial x}+\frac{\partial u}{\partial z}
\end{array}\right\}
$$

(3)Physical equation (generalized Hooke theorem) ${ }^{[9]}$ :

$$
\left.\begin{array}{l}
\varepsilon_{x}=\frac{1}{E}\left(\sigma_{x}-v\left(\sigma_{y}+\sigma_{z}\right)\right) ; \gamma_{x y}=\frac{2(1+v)}{E} \tau_{x y} \\
\varepsilon_{y}=\frac{1}{E}\left(\sigma_{y}-v\left(\sigma_{x}+\sigma_{z}\right)\right) ; \gamma_{y z}=\frac{2(1+v)}{E} \tau_{y z} \\
\varepsilon_{x}=\frac{1}{E}\left(\sigma_{z}-v\left(\sigma_{x}+\sigma_{y}\right)\right) ; \gamma_{z x}=\frac{2(1+v)}{E} \tau_{z x}
\end{array}\right\}
$$

Where, $E$ is modulus of elasticity, $v$ is Poisson's ratio; $u 、 v 、 w$ is the displacement in the direction of $x, y, z ; \varepsilon_{x}, \varepsilon_{y}, \varepsilon_{z}$ is the strain in the direction of $x, y, z ; \delta_{x}, \delta_{y}, \delta_{z}$ is the stress in the direction of $x, y, z ; \tau_{x y}, \tau_{y z}, \tau_{z x}$ is the shear stress in the direction of $x y, y z, z x ; f_{x}, f_{y}, f_{z}$ is inertia force in the direction of $x, y, z$.

\section{Finite element model of porcelain insulator}

A three dimensional finite element model of porcelain insulator is established by ANSYS software. As the structure of the porcelain insulator is more symmetrical, the volume is relatively large, so the model is simplified to six parts. The finite element model, from top to bottom as follows: at the top of the gossan, palm connection, flange, umbrella skirt, flange, base. Porcelain post insulator of the 
partial ball socket of connection structure size is standard, and it is not the study object, so you can simplify the gossan structure and in the analysis of simulation results is not considered in the gossan of deformation.

In this paper, the voltage level of the porcelain insulator is $110 \mathrm{kV}$, the model is $\mathrm{ZSW}-126 / 6$, and the structural parameters are shown in Table 1.

Table $1110 \mathrm{kV}$ porcelain insulator structure parameters

\begin{tabular}{|c|c|}
\hline Overall structure height/mm & 1389 \\
\hline Umbrella skirt structure height/mm & 978 \\
\hline Umbrella skirt number & 14 \\
\hline Umbrella skirt diameter/mm & 120 \\
\hline $\begin{array}{c}\text { Sig umbrella: } 230 \\
\text { Small umbrella:200 }\end{array}$ \\
\hline $\begin{array}{c}\text { Diameter between upper flange and the first } \\
\text { piece of skirt/mm }\end{array}$ & 120 \\
\hline $\begin{array}{c}\text { Diameter between lower flange and the first } \\
\text { piece of skirt/mm }\end{array}$ & 135 \\
\hline
\end{tabular}

Finite element model of porcelain column insulator is shown in Fig.2.

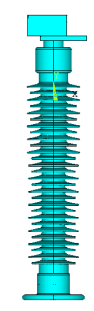

Fig. 2 Finite element model of $110 \mathrm{kV}$ porcelain insulator.

The gossip, palm, flange connection is made of cast iron material, the umbrella skirt is made of ceramic material, and the lower flange and the base uses cement materials, and porcelain pieces are fixed in the upper and lower flanges. Because the cast iron flange is mainly made of ductile iron, and the size of a large margin, with a great mechanical strength, so in the stress simulation calculation don't have to consider the role of flange plus strong tendons.

The length unit is $\mathrm{mm}$, force load unit is $\mathrm{N}$, stress unit is $\mathrm{MPa}$. The main performance parameters of the related materials are shown in Table 2.

Table 2 Main performance parameters of material

\begin{tabular}{|c|c|c|c|}
\hline Parameter & Porcelain & Cast iron & Cement \\
\hline Modulus of elasticity/pa & $9.47 \times 10^{10}$ & $1.75 \times 10^{11}$ & $3.1 \times 10^{10}$ \\
\hline Poisson's ratio & 0.16 & 0.28 & 0.215 \\
\hline Mass density $\left(\mathrm{kg} / \mathrm{m}^{3}\right)$ & 2600 & 7800 & 2300 \\
\hline
\end{tabular}

Establish the model, the corresponding material parameters and element parameters are given, then mesh division of the model will be carried out. As the main component of the porcelain insulator is cast iron, ceramic and cement, so the finite element model can be divided into three parts: the upper structure, the umbrella skirt structure, the lower structure

Therefore, in order to meet the requirements of each part of the grid division, and after two 
grid method, finally free grid method is determined to use. Grid cell number is $2,309,781$, node number is 450,256. The overall grid shape is regular, so the convergence rate is fast and the accuracy of the results will be higher. Its finite element model parameters are set as shown in Table 3 ,

Table 3 Finite element model of $110 \mathrm{kV}$ porcelain insulator

\begin{tabular}{|c|c|c|c|c|}
\hline & $\begin{array}{c}\text { Texture of } \\
\text { material }\end{array}$ & Mesh size/mm & Grid cell & node \\
\hline Gossan & \multirow{3}{*}{ CAST IRON } & 0.006 & \multirow{6}{*}{2309781} & \multirow{6}{*}{450256} \\
\hline Junction palm & & 0.0025 & & \\
\hline Upper flange & & 0.005 & & \\
\hline $\begin{array}{l}\text { Umbrella skirt } \\
\text { structure }\end{array}$ & PORCELAIN & 0.0045 & & \\
\hline Lower flange & \multirow{2}{*}{ CEMENT } & 0.006 & & \\
\hline Base & & 0.004 & & \\
\hline
\end{tabular}

And the corresponding integral partitioning results are shown in Fig. 3.

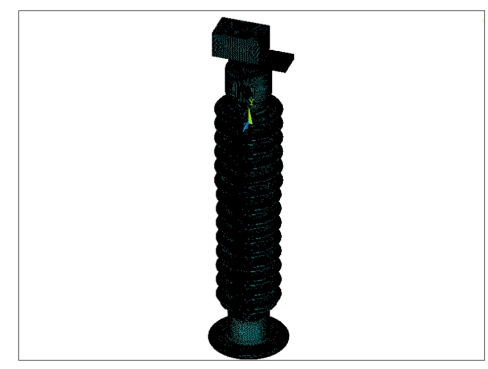

Fig. 3 Finite element mesh for porcelain insulator.

\section{The determination of empirical formula of stress strain and force load}

The stress calculation results of porcelain insulator under different load are calculated and the relationship between the stress and the load direction and the size of the porcelain insulator is obtained. In order to reflect the relationship between the stress and strain of the porcelain insulator and the different load, the stress and strain and the stress load should be summarized in order to obtain the corresponding relationship between the stress load and stress and strain.

The determination of the empirical formula of stress and load

Generally speaking, the empirical formula is concluded on the basis of enough theoretical calculation results. Therefore, according to the curve of the curve in the MATLAB to make the corresponding data fitting, can get the fitting formula and the corresponding parameters to determine the most real equation.

Relationship equation of stress maximum value of porcelain insulator is: 
$\sigma_{1 \max }=0.0264 F-0.017$

The maximum stress and load equation of the umbrella skirt is:

$\sigma_{1 \max }^{\prime}=0.0055 F-0.015$

In Eq. 11, Eq. 12, $\mathrm{F}$ is the value of the force load and its unit is $\mathrm{N}$.

SSE is used to characterize the fit of the data and the original data in general fitting equation. The statistical parameter is the sum of the squares of the errors of the fitting data and the corresponding points of the raw data. Close to $0 \mathrm{SEE}$ is, the fitting equation is more accurate, and the prediction of data is more successful. Eq. 11 variance is 0.0023 , Eq. 12 variance is 0.002 whose degree of accuracy is higher, and the fitting degree of the real data is higher.

The determination of empirical formula of strain and load

In order to determine the relationship between the strain and the load, the strain data of the porcelain insulator under different loads is concluded. The strain data corresponding to the load of the porcelain insulator in the $50 \mathrm{~N} \sim 300 \mathrm{~N}$ are simulated and calculated, and the results are shown in Table 4:

Table 4 Strain Data under Different Loads of Porcelain Post Insulator in $45^{\circ}$

\begin{tabular}{|c|c|c|}
\hline load value/N & $\varepsilon_{1 \max }$ & $\varepsilon_{1 \mathrm{max}}$ \\
\hline 50 & $0.622 \times 10^{-5}$ & $0.225 \times 10^{-5}$ \\
\hline 80 & $0.995 \times 10^{-5}$ & $0.359 \times 10^{-5}$ \\
\hline 100 & $0.124 \times 10^{-4}$ & $0.449 \times 10^{-5}$ \\
\hline 120 & $0.149 \times 10^{-4}$ & $0.539 \times 10^{-5}$ \\
\hline 150 & $0.186 \times 10^{-4}$ & $0.674 \times 10^{-5}$ \\
\hline 180 & $0.224 \times 10^{-4}$ & $0.809 \times 10^{-5}$ \\
\hline 200 & $0.249 \times 10^{-4}$ & $0.898 \times 10^{-5}$ \\
\hline 220 & $0.274 \times 10^{-4}$ & $0.998 \times 10^{-5}$ \\
\hline 240 & $0.298 \times 10^{-4}$ & $0.108 \times 10^{-4}$ \\
\hline 250 & $0.311 \times 10^{-4}$ & $0.112 \times 10^{-4}$ \\
\hline 270 & $0.336 \times 10^{-4}$ & $0.121 \times 10^{-4}$ \\
\hline 300 & $0.373 \times 10^{-4}$ & $0.135 \times 10^{-4}$ \\
\hline 400 & $0.497 \times 10^{-4}$ & $0.180 \times 10^{-4}$ \\
\hline
\end{tabular}

Where, $\varepsilon_{1 \max } \longrightarrow$ Maximum strain of insulator, $\varepsilon_{1 \max }^{\prime}$-Maximum strain of umbrella skirt.

Relationship equation of strain maximum value of porcelain insulator strain is:

$\varepsilon_{1 \max }=1.25 \times 10^{-7} \mathrm{~F}-3.4 \times 10^{-8}$

Relationship equation of strain maximum and force load of umbrella skirt is:

$\varepsilon_{1 \max }^{\prime}=4.48 \times 10^{-8} F+1.5 \times 10^{-8}$

In the Eq.13, Eq.14, the variance are $1.155 \times 10^{-14}, 3.43 \times 10^{-15}$ respectively. When the variance is small enough within the allowable error range, it can be considered as 0 , and its accuracy is high enough. So the above Eq.13 and Eq.14 have high fitting degree of real condition.

For the whole insulator, the main materials are cast iron, ceramic and cement, it can be said that the whole of the insulator is a nonlinear material properties, and the relationship between the stress and strain of the insulator umbrella skirt should meet Hooke's law, its approximate calculation is:

$$
\varepsilon_{1 \max }^{\prime \prime}=\frac{0.0055}{E}=\frac{0.0055}{1.18 \times 10^{5}}=4.66 \times 10^{-8}
$$

Error calculation: 


$$
\delta=\frac{4.66-4.48}{4.48} \times 100 \%=4 \%
$$

Because the stress value of the porcelain insulator is relatively large, the error is in the allowable range, which can be directly verified by the accuracy of the simulation results.

According to the fitting stress, strain and load relationship of the curves, the following Table 5 shows:

Table 5 Relationship between stress and strain of $110 \mathrm{kV}$ porcelain insulator

\begin{tabular}{|c|c|c|c|c|c|}
\hline & $0^{\circ}$ & $30^{\circ}$ & $45^{\circ}$ & $60^{\circ}$ & $90^{\circ}$ \\
\hline$s_{1}$ & $0.0386 \mathrm{~F}+0.002$ & $0.0328 \mathrm{~F}+0.004$ & $0.0264 \mathrm{~F}-0.017$ & $0.0182 \mathrm{~F}-0.002$ & $\underset{17}{0.0027 \mathrm{~F}-5.8 \times 10^{-}}$ \\
\hline$s_{1}^{\prime}$ & $0.0079 F+0.0008$ & $0.0068 F-0.0018$ & $0.0055 \mathrm{~F}-0.015$ & $0.0038 F-0.0038$ & $\underset{17}{0.0005 \mathrm{~F}-1.5 \times 10^{-}}$ \\
\hline$e_{1}$ & $\begin{array}{c}1.83 \times 10^{-7} \mathrm{~F}- \\
2 \times 10^{-9}\end{array}$ & $\begin{array}{c}1.55 \times 10^{-} \\
{ }^{7} \mathrm{~F}+1 \times 10^{-8}\end{array}$ & $\begin{array}{c}1.25 \times 10^{-7} \mathrm{~F}- \\
3 \times 10^{-8}\end{array}$ & $\begin{array}{c}8.53 \times 10^{-} \\
{ }^{7} \mathrm{~F}+3 \times 10^{-9}\end{array}$ & $\begin{array}{c}1.26 \times 10^{-8} \mathrm{~F}- \\
4 \times 10^{-9}\end{array}$ \\
\hline$e_{1}$ & $\begin{array}{c}6.47 \times 10^{-8} \mathrm{~F}- \\
1 \times 10^{-9}\end{array}$ & $5.56 \times 10_{8}^{-8}-1 \times 10^{-}$ & $\begin{array}{c}4.48 \times 10^{-8} \mathrm{~F}- \\
1 \times 10^{-8}\end{array}$ & $\begin{array}{c}3.13 \times 10^{-} \\
{ }^{8} \mathrm{~F}+5 \times 10^{-9}\end{array}$ & $\begin{array}{c}4.56 \times 10^{-} \\
{ }^{9} \mathrm{~F}+2 \times 10^{-9}\end{array}$ \\
\hline
\end{tabular}

\section{Conclusion}

(1) This paper summarizes the present methods of fracture evaluation, and concludes the advantages of the proposed method in this paper. The advantage is that the stress distribution of the insulator can be achieved by the detection of the real-time tension of the porcelain insulator, and can really realize the nondestructive testing of the porcelain insulator.

(2) Through the analysis of the structural characteristics of the ceramic material, the fracture mode is open. Applying the linear elastic fracture mechanics, the fracture process of the insulator is analyzed, and the brittle fracture theory of the porcelain insulator is summarized. The main reason of the brittle fracture of the porcelain insulator is that the connection is not normal.

(3) The finite element model of porcelain insulators with different voltage levels is established, simulation calculation of the model is carried out by the force load in different directions. The stress distribution of the porcelain insulator under different directions is obtained which indicates that the greater the tension of the wire drawing of the post bar of the porcelain insulator, the more conducive to the safe operation of insulators, relatively speaking.

(4) The stress distribution of the porcelain insulator under different loads is calculated by using the force load of different sizes. The concrete results are as follows: when the load is in a certain direction, the stress concentration at the root of the umbrella is the most severe at the bottom. And the maximum value of stress is increasing with the increase of the load, which is a linear relationship.

\section{References}

[1] Ma Mingye, Li Yan.Failure analysis of post insulator and porcelain bushing and the research of ultrasonic inspection technology[J]. Ningxia electric power, 2007(3):52-57. In Chinese

[2] Su Zhiyi, Ju wenjun. Damage cause and detection of insulator in power network operation[J]. Electric power equipment, 2005, 6(3):10-13. In Chinese

[3] Liu Hui. Status quo and development trend of line insulator products at home and abroad[J]. Science and Technology Innovation Herald, 2007,(26):97.In Chinese 
[4] Gong Jianghong. Fracture mechanics of ceramic materials. Beijing: Tsinghua University press, 2001. In Chinese

[5] G.R.Irwin. Handbuch der Physick.Vol.6, Springer, Berlin, 551(1958).

[6] Yu Maohong, Zan Yuewen. Development and Prospect of strength theory[J]. Engineering Mechanics, 2004, 21(6):1-18. In Chinese

[7] Zyczkowski M. Combined loadings in the theory of plasticity [M]. Polish Scientific Publishers, 1981.

[8] Wang Kailong, Xu Hong. Application of ANSYS finite element analysis system in nonlinear analysis of structures[A]. Beijing: North China Electric Power University press, 2006: 787-791. In Chinese

[9] Jiang Yuchuan, Zhang Jianhai, Li akimasa. Elastic mechanics and finite element method [M]. Beijing: Science Press, 2006. In Chinese

[10] ANSYS Element Referenee, ANSYSIne.ANSYS6.1, 2001. 